\title{
Laparoscopic versus open hemihepatectomy: comprehensive comparison of complications and costs at 90 days using a propensity method
}

\author{
Francisco Riquelme ${ }^{1}$. César Muñoz ${ }^{1} \cdot$ Fabio Ausania $^{1}$ - Amelia J. Hessheimer ${ }^{1}$. Ferrán Torres ${ }^{2} \cdot$ David Calatayud $^{1}$. \\ Raffaele Sandomenico ${ }^{1}$. Rocío García Pérez ${ }^{1}$. Joana Ferrer ${ }^{1}$. José Fuster ${ }^{1}$. Juan Carlos García-Valdecasas ${ }^{1}$. \\ Constantino Fondevila ${ }^{1,3}$
}

Received: 19 May 2020 / Accepted: 21 July 2020 / Published online: 30 July 2020

(c) The Author(s) 2020

\begin{abstract}
Laparoscopic hemihepatectomy (LHH) may offer advantages over open hemihepatectomy (OHH) in blood loss, recovery, and hospital stay. The aim of this study is to evaluate our recent experience performing hemihepatectomy and compare complications and costs up to 90 days following laparoscopic versus open procedures. Retrospective evaluation of patients undergoing hemihepatectomy at our center 01/2010-12/2018 was performed. Patient, tumor, and surgical characteristics; 90-day complications; and costs were analyzed. Inverse probability of treatment weighting (IPTW) was used to balance covariates. A total of 141 hemihepatectomies were included: $96 \mathrm{OHH}$ and $45 \mathrm{LHH}$. While operative times were longer for LHH, blood loss and transfusions were less. At 90 days, there were similar rates of liver-specific and surgical complications but fewer medical complications following LHH. Medical complications that arose with greater frequency following $\mathrm{OHH}$ were primarily pulmonary complications and urinary and central venous catheter infections. Complications at 90 days were lower following LHH (Clavien-Dindo grade $\geq$ III OHH 23\%, LHH 11\%, $p=0.130$; Comprehensive Complication Index OHH $20.0 \pm 16.1$, LHH $10.9 \pm 14.2, p=0.001$ ). While operating costs were higher, costs for hospital stay and readmissions were lower with LHH. Patients undergoing LHH experience a significant reduction in postoperative medical complications and costs, resulting in 90-day cost equity compared with $\mathrm{OHH}$.
\end{abstract}

Keywords Hemihepatectomy · Laparoscopy · Major liver resection · Comprehensive complication index

Constantino Fondevila

cfondevila@ub.edu

1 Hepatopancreatobiliary Surgery and Transplantation, General and Digestive Surgery Service, Hospital Clínic, IDIBAPS, CIBERehd, University of Barcelona, Barcelona, Spain

2 Medical Statistics Core Facility, Faculty of Medicine, IDIBAPS, Hospital Clínic Barcelona, Barcelona, Spain, and Biostatistics Unit, Universidad Autónoma de Barcelona, Barcelona, Spain

3 Department of General and Digestive Surgery, Hospital Clínic, University of Barcelona, C/ Villarroel 170, 08036 Barcelona, Spain

\section{Introduction}

The laparoscopic approach to abdominal interventions better preserves the integrity and function of the abdominal wall and limits exposure and manipulation of abdominal viscera, not only leading to improved cosmesis but also reduced intraabdominal adhesions and wound and wall complications. The loss of manual tissue manipulation and control is compensated, at least to some degree, by magnified and direct visualization. In the field of hepatic surgery, the results of clinical retrospective studies, meta-analyses, and one randomized trial indicate that the aforementioned advantages may translate into less perioperative blood loss and improved recovery, with decreased postoperative complications and hospital stay [1-4]. For these reasons, the laparoscopic approach is now considered standard-of-care for the resection of lesions in the left lateral and anterior hepatic segments [5]. 
In contrast with the aforementioned minor liver resections, laparoscopic hemihepatectomy (LHH) is still considered an advanced technique to only be carried out by experienced surgeons. While transection may proceed across hepatic anatomical planes, these planes remain intraparenchymal, highly vascularized, and not recognized by standard traction-countertraction maneuvers. As well, vascular and biliary structures to one side of the liver have to be ligated while preserving structures on the contralateral side, and the liver has to be separated to a certain extent from the inferior vena cava, intraoperative injury to which can be devastating. Accordingly, a learning curve of at least 55 cases for right hemihepatectomy after prior experience with minor laparoscopic liver resections has been suggested $[5,6]$.

Aside from increased complexity, cost associated with LHH can also be increased, in particular related to the use of more expensive surgical instruments and devices and longer operative times versus open hemihepatectomy (OHH). Cost analyses have determined that operative costs may be significantly higher for $\mathrm{LHH}$ than for $\mathrm{OHH}$, though the increase in upfront cost may be offset, at least in part, by improved and less costly postoperative recovery and hospital stay [7-10].

The laparoscopic approach to hepatic resection was introduced at our center in 2005 , and we currently perform approximately $75 \%$ of our hepatectomies laparoscopically. Based on the results of previous studies, we hypothesized that fewer complications arise after LHH and this difference may translate into not only a shorter and less costly postoperative stay but also fewer readmissions and ongoing complications through the end of the third postoperative month, whereby the great majority of complications following major hepatectomy has occurred [11]. The aim of the present study is, therefore, to comprehensively evaluate the recent years of our experience performing hemihepatectomy and to compare cumulative complications and costs measured up to 90 days following laparoscopic versus open procedures.

\section{Patients and methods}

\section{Study design}

This study is a retrospective evaluation of patients undergoing right or left hemihepatectomy at our center between January 2010 and December 2018. Exclusion criteria included extended hepatectomy, additional biliary or vascular resection, previous portal vein embolization, previous hepatectomy (including two-stage hepatectomy), emergency hepatectomy, living donor hepatectomy, and synchronous multiorgan resection (including bowel resection). Approval for the study was obtained from our Institution's Committee on Ethics in Medical Research prior to initiating data analysis (study number HCB/2018/1179), which waived the need for written consent.

\section{Preoperative evaluation}

Preoperatively, all patients underwent routine laboratory tests and contrast-enhanced multislice abdominal computed tomography $(\mathrm{CT})$ and and/or gadolinium-enhanced hepatic magnetic resonance imaging (MRI) and were discussed in multidisciplinary tumor board. For colorectal liver metastases, preoperative chemotherapy was indicated in cases of initially unresectable disease until conversion to resectability, borderline resectable disease, and initially resectable disease with synchronous presentation. Initial resection of the primary tumor was performed in cases with synchronous presentation.

\section{Surgical procedures}

All surgeons performing LHH had previously performed a minimum of 30 laparoscopic liver resections, and all surgeons performing both $\mathrm{LHH}$ and $\mathrm{OHH}$ had extensive experience performing open hepatic surgery.

\section{Laparoscopic hemihepatectomy}

The patient was placed with legs spread and in reverse Trendelenburg. Five or six ports were used, with the $12-\mathrm{mm}$ camera port placed along the anterior axillary line. Pneumoperitoneum was maintained $12-14 \mathrm{mmHg}$. Following mobilization of the right or left liver from its ligamentous attachments, intraoperative ultrasound was performed to confirm the number and size of the lesion(s). Preparation for intermittent Pringle maneuver was performed by encircling the hepatic hilum with a tourniquet, which was exteriorized through a thoracostomy tube inserted through the abdominal wall above the pubic symphysis. An extrahepatic intraglissonian pedicle approach was used to dissect and encircle the ipsilateral hepatic artery and portal vein. For left LHH, the left lobe was pulled upward, and Arantius' ligament approach was used to encircle the left hepatic vein [12]. For right LHH, retrohepatic veins and the hepatocaval ligament were divided between titanium or Hem-o-lok® clips, followed by dissection and encircling of the right hepatic vein to perform liver hanging maneuver. Ischemic delimitation of the transection plane was achieved after pedicle clamping, and liver transection was performed using a combination of LigaSure $^{\mathrm{TM}}$ (Medtronic, Minneapolis, Minnesota, USA) and 
CUSA $®$ Excel (Valleylab, Boulder, Colorado, USA). Vascular staplers were used to transect both the hepatic pedicles at the level of the hilar plate and hepatic veins and the end of parenchymal transection. The surgical specimen was placed in an Endo Catch ${ }^{\mathrm{TM}}$ Specimen Retrieval Pouch (Medtronic, Minneapolis, Minnesota, USA) and removed through a Pfannenstiel incision. The incision was then closed, intraabdominal hemostasis was checked, and an abdominal drain was placed.

\section{Hybrid hemihepatectomy}

The hybrid technique was defined as a planned procedure that started with liver mobilization and isolation of hepatic pedicle through laparoscopic approach. Thereafter, parenchymal transection was performed through a 6-8 cm upper midline laparotomy, which was also used for specimen extraction.

\section{Open hemihepatectomy}

Open approach was performed using a "J"-shaped or right subcostal incision with subxiphoid extension. After exploration of the abdominal cavity and hemiliver mobilization, intraoperative ultrasound was performed. The hepatic hilum was encircled with a tourniquet to allow subsequent intermittent Pringle maneuver. The ipsilateral hepatic artery and portal vein were then dissected and clamped in the hepatic hilum, delimiting the transection plane. CUSA ${ }^{\circledR}$ was used for parenchymal transection. The hemiliver pedicle was sectioned between sutures and the suprahepatic vein using a vascular stapling device. An abdominal drain was routinely placed.

\section{Definition of variables and outcomes}

The main objective of the study was to assess clinical outcomes and costs associated with LHH and $\mathrm{OHH}$ up to 90 days postoperatively. Cases of LHH that resulted in conversion to open surgery were not censored and were included among the LHH group for all analyses based on intention-to-treat. The following patient-related variables were recorded: age, sex, body mass index (BMI), American Society of Anesthesiologists (ASA) class, and previous abdominal surgery (open or laparoscopic surgery or percutaneous radiofrequency ablation). Co-morbid conditions were quantified according to the age-adjusted Charlson comorbidity index, which was calculated excluding liver tumor variables [13]. Liver-specific variables that were recorded included principle diagnosis and history of liver disease. Tumor-specific variables included the number, size, extent, and distribution of lesions and history of preoperative chemotherapy. Intraoperative variables included associated procedures (e.g., non-anatomical resection or radiofrequency ablation in the contralateral hemiliver), the use and duration of intermittent Pringle maneuver, operative time, blood loss, and reasons for conversion to open surgery in the LHH group. Surgical pathology was evaluated for tumor size and margins, with resection margins distancing $\leq 1 \mathrm{~mm}$ from tumor cells classified as "R1". Postoperative outcomes that were recorded included the need for blood transfusion, postoperative intensive care stay, postoperative hospital stay, complications, re-interventions, unplanned readmissions, and mortality. All complications occurring up to 90 days postoperatively were recorded and graded according to the Clavien-Dindo [14] and Comprehensive Complication Index (CCI) systems, the latter of which computes all the complications a patient experiences in an index ranging from 0 to 100 [15]. Postoperative complications were categorized as surgical, medical, or liver specific. Liver-specific complications were classified according to the International Study Group for Liver Surgery (ISGLS) criteria. Bile leak was defined as a drain bilirubin concentration $>3$-times higher than the serum bilirubin concentration at or beyond the third postoperative day and graded as A-requiring no change in patient's clinical management, $\mathrm{B}$-requiring therapeutic intervention but manageable without surgical re-intervention, or $\mathrm{C}$-requiring surgical re-intervention [16]. Post-hepatectomy liver failure (PHLF) was defined as increased international normalized ratio (INR) and hyperbilirubinemia at or beyond the fifth postoperative day and graded as A-analytical deterioration not requiring any change in management, B-deviation from regular postoperative clinical pathway managed without invasive treatment, or C-PHLF requiring an invasive procedure [17]. Postoperative ascites was defined as drain fluid output $>10 \mathrm{~mL} / \mathrm{kg} / \mathrm{day}$ at or beyond the third postoperative day [18]. In addition to the hospital electronic medical record, the regional shared electronic medical record was checked for readmissions in other centers.

\section{Costs}

Intraoperative, postoperative, and readmission costs were obtained, including variable and fixed costs that were charged in each case. Intraoperative costs included consumables (anesthetic drugs, transfusions, surgical instrumentation, and devices) and operating room and staff costs. Postoperative costs included pharmacy, transfusions, ICU and/or floor beds, studies and procedures (radiological, endoscopic, and/or surgical), and other miscellaneous expenditures (laboratory, nursing, and other professional care). Readmission expenses including re-intervention 
were grouped under total readmission costs. Taking into account the study inclusion period (2010-2018), costs were adjusted according to the mean annual inflation rate: $2010+1.8 \%, 2011+3.2,2012+2.44,2013+1.42$, $2014-0.15,2015-0.5,2016-0.2,2017+2.97$, $2018+1.67[19]$.

\section{Data and statistical analysis}

Categorical variables are described as frequencies and percentages and continuous variables as mean \pm standard deviation or median (25-75\% interquartile range). The propensity score method, which simulates the effects of a randomized trial for observational data, was used to estimate study outcomes. Inverse probability of treatment weighting (IPTW) of the propensity scores was used to create a pseudo-population in which study groups were balanced across covariates using data blinded to outcomes. Weights were derived using logistic regression to estimate average treatment effects in treated patients and stabilized by treatment prevalence. The following covariates were included in the propensity models: age, sex, ASA classification, BMI, age-adjusted Charlson comorbidity index, previous abdominal surgery, previous radiofrequency ablation, cirrhosis, indication for surgery, number of liver lesions, diameter of the largest lesion, number of affected liver segments, unilobar versus bilobar liver disease, preoperative chemotherapy and number of cycles, and planned additional procedure. Categorical variables were compared using the Chi-square test and continuous variables using ANOVA with rank-transformed data for both raw and IPTW-adjusted analyses. Covariate balance was assessed using the standardized difference, which is the difference between groups divided by the pooled standard deviation. Statistical significance was defined as $p<0.05$. Statistical analyses were performed using SAS 9.4 (SAS Institute, Inc., Cary, North Carolina, USA).

Table 1 Patient, tumor, and surgical characteristics used for the propensity model

\begin{tabular}{|c|c|c|c|c|c|c|c|c|}
\hline & \multicolumn{4}{|l|}{ Raw analysis } & \multicolumn{4}{|c|}{ IPTW analysis } \\
\hline & OHH $(n=96)$ & LHH $(n=45)$ & $p$ value & $\begin{array}{l}\text { Standard- } \\
\text { ized differ- } \\
\text { ence }\end{array}$ & $\mathrm{OHH}$ & LHH & $p$ value & $\begin{array}{l}\text { Standardized } \\
\text { difference }\end{array}$ \\
\hline Age (years) & 65 [57-72] & 65 [55-69] & 0.476 & -0.120 & 65 [57-72] & 67 [57-69] & 0.889 & 0.028 \\
\hline Sex female & $38(40 \%)$ & $23(51 \%)$ & 0.197 & -0.233 & $42(45 \%)$ & $16(47 \%)$ & 0.872 & -0.032 \\
\hline Body mass index & 26 [23-28] & 25 [23-28] & 0.329 & -0.177 & 26 [23-27] & 25 [23-28] & 0.461 & -0.150 \\
\hline $\begin{array}{l}\text { Age-adjusted Charlson comorbidity } \\
\text { index }\end{array}$ & $2[1-4]$ & $2[1-3]$ & 0.406 & -0.153 & $2[2,3]$ & $2[1-3]$ & 0.669 & -0.087 \\
\hline \multicolumn{9}{|l|}{ ASA class } \\
\hline I & $4(4 \%)$ & $4(9 \%)$ & 0.258 & 0.192 & $4(4 \%)$ & $2(5 \%)$ & 0.872 & 0.032 \\
\hline II & $71(74 \%)$ & $34(76 \%)$ & 0.839 & 0.037 & $70(76 \%)$ & $27(77 \%)$ & 0.885 & 0.029 \\
\hline III & $21(22 \%)$ & $7(16 \%)$ & 0.380 & -0.163 & $19(20 \%)$ & $6(18 \%)$ & 0.810 & -0.048 \\
\hline Previous abdominal procedure & $81(84 \%)$ & $38(84 \%)$ & 0.991 & 0.002 & $80(86 \%)$ & $30(87 \%)$ & 0.866 & 0.034 \\
\hline Radiofrequency ablation & $3(3 \%)$ & 0 & 0.230 & -0.254 & $2(2 \%)$ & $0(0 \%)$ & 0.378 & -0.212 \\
\hline Open surgery & $42(44 \%)$ & $9(20 \%)$ & 0.006 & -0.527 & $37(39 \%)$ & $10(29 \%)$ & 0.276 & -0.221 \\
\hline Laparoscopic surgery & $39(41 \%)$ & $29(64 \%)$ & 0.008 & 0.491 & $43(46 \%)$ & $20(58 \%)$ & 0.244 & 0.233 \\
\hline Cirrhosis & $3(3 \%)$ & $2(4 \%)$ & 0.692 & 0.069 & $3(3 \%)$ & $1(3 \%)$ & 0.973 & 0.007 \\
\hline Malignant disease & $89(93 \%)$ & $38(84 \%)$ & 0.126 & 0.262 & $85(91 \%)$ & $30(87 \%)$ & 0.455 & 0.142 \\
\hline Preoperative chemotherapy & $44(46 \%)$ & $29(64 \%)$ & 0.039 & 0.381 & $49(52 \%)$ & $21(61 \%)$ & 0.374 & 0.178 \\
\hline Multiple lesions & $49(51 \%)$ & $26(58 \%)$ & 0.454 & 0.136 & $52(56 \%)$ & $19(55 \%)$ & 0.890 & -0.027 \\
\hline Number of lesions & $2[1-4]$ & $2[1-3]$ & 0.903 & 0.023 & $2[1-4]$ & $2[1-4]$ & 0.593 & 0.109 \\
\hline Number of affected segments & $2[1-3]$ & $2[2,3]$ & 0.587 & 0.100 & $2[2,3]$ & $3[2,3]$ & 0.643 & 0.095 \\
\hline Maximum diameter of lesions (mm) & $33[22-55]$ & $27[15-45]$ & 0.114 & -0.283 & 33 [22-55] & $32[15-45]$ & 0.436 & -0.155 \\
\hline Bilobar disease & $26(27 \%)$ & $11(24 \%)$ & 0.739 & -0.060 & $23(25 \%)$ & $10(29 \%)$ & 0.683 & 0.080 \\
\hline Planned associated procedure & $30(31 \%)$ & $10(22 \%)$ & 0.267 & -0.205 & $27(29 \%)$ & $9(26 \%)$ & 0.798 & -0.051 \\
\hline
\end{tabular}

Descriptive statistics are frequencies (\%) for categorical variables and median [25-75\% interquartile range]. Bold marked figures are for $p<0.05$ ASA American Society of Anesthesiologists, IPTW inverse probability of treatment weighting, LHH laparoscopic hemihepatectomy, $O H H$ open hemihepatectomy 


\section{Results}

Between 01/2010 and 12/2018, 838 liver resections were performed at our center. Based on inclusion-exclusion criteria, 141 were included for analysis: $96 \mathrm{OHH}$ and $45 \mathrm{LHH}$ (70\% and 64\% right hemihepatectomies, respectively).

\section{Patient demographics and tumor and surgical characteristics}

Baseline patient demographical information and tumor and surgical characteristics are listed in Table 1 . The average patient in this study was around 65 years of age and overweight (BMI 25-26), with an ASA score of II. Men and women were equally represented among included patients. The indication for hemihepatectomy was malignant tumor in $90 \%$ of cases (OHH: $71 \%$ metastases, $22 \%$ primary liver tumors; LHH: $76 \%$ metastases, $9 \%$ primary liver tumors); consequentially, the majority of patients had a history of preoperative chemotherapy. In terms of tumor characteristics, the median number was two lesions affecting $\geq 2$ liver segments, and the median size of the largest lesion was approximately $3 \mathrm{~cm}$. Approximately a quarter of patients presented bilobar disease that required an associated procedure in addition to hemihepatectomy. On raw analysis, a greater percentage of patients in the LHH group had previously received chemotherapy and undergone laparoscopic abdominal procedures as opposed to open ones. Following
IPTW adjustments, covariates were balanced between the two groups, with all $p$ values $<0.05$ and standardized differences $<0.25$.

\section{Operative characteristics and intraoperative outcomes}

Table 2 lists variables associated with the surgical interventions and intraoperative outcomes. Rates of any associated intraoperative procedure were similar between the two groups (29\% OHH, 26\% LHH, $p=0.798$ ). However, when analyzing associated procedures individually, more patients in the LHH group underwent concomitant radiofrequency ablation $(2 \% \mathrm{OHH}, 14 \% \mathrm{LHH})$, while more patients in the $\mathrm{OHH}$ group underwent concomitant non-anatomical resection in the remnant hemiliver $(24 \% \mathrm{OHH}, 12 \% \mathrm{LHH}$, $p=0.010$ ). As well, more patients in the LHH group were subjected to intermittent Pringle maneuver (44\% OHH, $84 \%$ LHH, $p<0.001$ ).

In the LHH group, approach was purely laparoscopic in 35 cases (78\%) and hybrid in $10(22 \%)$. All hybrid cases were performed during the first 3 years of the study period. Among pure laparoscopic cases, conversion to open surgery was performed in six (17\%): three for right upper quadrant adhesions, two for bleeding, and one for technical failure of the laparoscopic CUSA ${ }^{\circledR}$ device.

Operative times were significantly longer by $1 \mathrm{~h}$ in the LHH group (210 [180-255] minutes OHH, 270 [225-335] minutes LHH, $p<0.001$ ), but LHH intraoperative blood

Table 2 Operative variables and outcomes

\begin{tabular}{|c|c|c|c|c|c|c|}
\hline & \multicolumn{3}{|l|}{ Raw analysis } & \multicolumn{3}{|l|}{ IPTW analysis } \\
\hline & $\mathrm{OHH}(n=96)$ & LHH $(n=45)$ & $p$ value & $\mathrm{OHH}$ & LHH & $p$ value \\
\hline Right hepatectomy & $67(70 \%)$ & $29(64 \%)$ & 0.526 & $72 \%$ & $61 \%$ & 0.237 \\
\hline Associated procedure (any) & $30(31 \%)$ & $10(22 \%)$ & 0.268 & $29 \%$ & $26 \%$ & 0.798 \\
\hline Non-anatomical resection & $24(25 \%)$ & $5(11 \%)$ & 0.018 & $24 \%$ & $12 \%$ & 0.010 \\
\hline Radiofrequency ablation & $2(2 \%)$ & $5(11 \%)$ & & $2 \%$ & $14 \%$ & \\
\hline Other & $4(4 \%)$ & 0 & & $3 \%$ & 0 & \\
\hline Pringle maneuver & $42(46 \%)$ & $38(86 \%)$ & $<0.001$ & $44 \%$ & $84 \%$ & $<0.001$ \\
\hline Pringle maneuver (min) & 18 [15-37] & $33[20-45]$ & 0.028 & $17[15-36]$ & $30[15-40]$ & 0.081 \\
\hline Conversion to open surgery ${ }^{\mathrm{a}}$ & - & $6(17 \%)$ & - & - & - & - \\
\hline Bleeding & - & $2(6 \%)$ & - & - & - & - \\
\hline Adhesions & - & $3(9 \%)$ & - & - & - & - \\
\hline Technical failure ${ }^{b}$ & - & $1(3 \%)$ & - & - & - & - \\
\hline Operative time $(\min )$ & 210 [180-255] & 270 [225-340] & $<0.001$ & 210 [180-255] & 270 [225-335] & $<0.001$ \\
\hline Blood loss (mL) & 300 [200-500] & 200 [100-300] & 0.012 & $300[150-500]$ & 200 [50-300] & 0.018 \\
\hline $\mathrm{R} 1$ resection & $13(14 \%)$ & $5(11 \%)$ & 0.656 & $13(14 \%)$ & $4(11 \%)$ & 0.612 \\
\hline
\end{tabular}

Descriptive statistics are frequencies (\%) for categorical variables and median [25-75\% interquartile range]

$I P T W$ inverse probability of treatment weighting, $L H H$ laparoscopic hemihepatectomy, $O H H$ open hemihepatectomy

${ }^{\mathrm{a}}$ Calculated as a percentage of pure laparoscopic cases $(n=35)$

${ }^{\mathrm{b}}$ Failure of the laparoscopic CUSA ${ }^{\circledR}$ 
Table 3 Postoperative complications and outcomes evaluated up to 90 days postoperatively

\begin{tabular}{|c|c|c|c|c|c|c|}
\hline & \multicolumn{3}{|l|}{ Raw analysis } & \multicolumn{3}{|c|}{ IPTW analysis } \\
\hline & $\mathrm{OHH}(n=96)$ & LHH $(n=45)$ & $p$ value & $\mathrm{OHH}$ & LHH & $p$ value \\
\hline \multicolumn{7}{|l|}{ At discharge } \\
\hline Overall complications & $62(65 \%)$ & $19(42 \%)$ & 0.012 & $60 \%$ & $39 \%$ & $\mathbf{0 . 0 3 9}$ \\
\hline Clavien-Dindo I/II & $46(48 \%)$ & $15(33 \%)$ & 0.103 & $44 \%$ & $29 \%$ & 0.127 \\
\hline Clavien-Dindo $\geq$ III & $16(17 \%)$ & $4(9 \%)$ & 0.217 & $16 \%$ & $10 \%$ & 0.416 \\
\hline $\mathrm{CCI}^{\mathrm{a}}$ & $18.0 \pm 16.2$ & $10.7 \pm 18.3$ & 0.001 & $16.5 \pm 16.1$ & $9.7 \pm 14.2$ & 0.009 \\
\hline Overall postoperative stay (days) & 8 [711] & $6[5-7]$ & $<0.001$ & $8[7-10]$ & $6[5-7]$ & $<0.001$ \\
\hline ICU stay (days) & $3[1-4]$ & $1[0-3]$ & $<0.001$ & $3[1-4]$ & $1[0-2]$ & $<0.001$ \\
\hline Blood transfusion & $17(18 \%)$ & $1(2 \%)$ & 0.010 & $15 \%$ & $1 \%$ & 0.024 \\
\hline PRBC units & $0.36 \pm 0.92$ & $0.04 \pm 0.30$ & 0.010 & $0.32 \pm 0.87$ & $0.02 \pm 0.18$ & 0.018 \\
\hline \multicolumn{7}{|l|}{ At 90 days } \\
\hline Liver-specific complications ${ }^{\mathrm{b}}$ & $16(17 \%)$ & $6(13 \%)$ & 0.611 & $16 \%$ & $12 \%$ & 0.600 \\
\hline Bile leak (any) & $9(9 \%)$ & $4(9 \%)$ & 0.926 & $9 \%$ & $9 \%$ & 0.948 \\
\hline Grade A & $2(2 \%)$ & $1(2 \%)$ & 0.396 & $2 \%$ & $1 \%$ & 0.610 \\
\hline Grade B & $5(5 \%)$ & 0 & & $4 \%$ & 0 & \\
\hline Grade C & $2(2 \%)$ & $2(4 \%)$ & & $3 \%$ & $5 \%$ & \\
\hline Ascites & $5(5 \%)$ & $2(4 \%)$ & 0.846 & $5 \%$ & $3 \%$ & 0.685 \\
\hline Encephalopathy & $5(5 \%)$ & 0 & 0.119 & $4 \%$ & 0 & 0.211 \\
\hline ISGLS PHLF (any) & $50(52 \%)$ & $13(30 \%)$ & 0.010 & $53 \%$ & $34 \%$ & 0.053 \\
\hline Grade A & $41(43 \%)$ & $12(27 \%)$ & 0.062 & $45 \%$ & $32 \%$ & 0.213 \\
\hline Grade B & $8(8 \%)$ & $1(2 \%)$ & & $8 \%$ & $2 \%$ & \\
\hline Grade C & $1(1 \%)$ & 0 & & $1 \%$ & 0 & \\
\hline General complications & $54(56 \%)$ & $14(31 \%)$ & 0.005 & $53 \%$ & $28 \%$ & 0.010 \\
\hline Surgical complications & $23(24 \%)$ & $5(11 \%)$ & 0.075 & $22 \%$ & $12 \%$ & 0.199 \\
\hline Deep surgical site infection & $11(12 \%)$ & $3(7 \%)$ & 0.375 & $10 \%$ & $6 \%$ & 0.546 \\
\hline Intestinal obstruction & $2(2 \%)$ & $1(2 \%)$ & 0.958 & $2 \%$ & $4 \%$ & 0.459 \\
\hline Hemorrahge/hematoma & $8(8 \%)$ & 0 & 0.046 & $7 \%$ & 0 & 0.105 \\
\hline Superficial surgical site infection & $8(8 \%)$ & $1(2 \%)$ & 0.166 & $9 \%$ & $2 \%$ & 0.148 \\
\hline Medical complications & $37(39 \%)$ & $9(20 \%)$ & 0.029 & $37 \%$ & $16 \%$ & 0.022 \\
\hline Arrhythmia & $2(2 \%)$ & 0 & 0.330 & $2 \%$ & 0 & 0.443 \\
\hline Pneumonia & $11(12 \%)$ & $1(2 \%)$ & 0.067 & $10 \%$ & $1 \%$ & 0.084 \\
\hline Pleural effusion & $6(6 \%)$ & 0 & 0.087 & $6 \%$ & 0 & 0.134 \\
\hline Paralytic ileus & $12(13 \%)$ & $7(16 \%)$ & 0.620 & $11 \%$ & $14 \%$ & 0.688 \\
\hline Urinary tract infection & $6(6 \%)$ & 0 & 0.087 & $6 \%$ & 0 & 0.140 \\
\hline CVC infection & $8(8 \%)$ & 0 & 0.046 & $7 \%$ & 0 & 0.103 \\
\hline Pulmonary embolism & $2(2 \%)$ & $1(2 \%)$ & 0.958 & $3 \%$ & $1 \%$ & 0.477 \\
\hline Overall complications & $71(74 \%)$ & $23(51 \%)$ & 0.007 & $69 \%$ & $44 \%$ & 0.010 \\
\hline Clavien-Dindo I/II & $48(50 \%)$ & $18(40 \%)$ & 0.267 & $46 \%$ & $33 \%$ & 0.189 \\
\hline Clavien-Dindo $\geq$ III & $23(24 \%)$ & $5(11 \%)$ & 0.075 & $23 \%$ & $11 \%$ & 0.130 \\
\hline $\mathrm{CCI}^{\mathrm{a}}$ & $21.6 \pm 16.1$ & $12.7 \pm 18.3$ & $<0.001$ & $20.0 \pm 16.1$ & $10.9 \pm 14.2$ & 0.001 \\
\hline Re-interventions at 90 days & $6(6 \%)$ & $2(4 \%)$ & 0.666 & $6 \%$ & $5 \%$ & 0.758 \\
\hline Readmissions at 90 days & $17(18 \%)$ & $3(7 \%)$ & 0.080 & $17 \%$ & $4 \%$ & 0.055 \\
\hline Mortality at 90 days & 0 & $1(2 \%)$ & 0.143 & 0 & $1 \%$ & 0.340 \\
\hline
\end{tabular}

Descriptive statistics are frequencies (\%) for categorical variables and mean \pm standard deviation or median [25-75\% interquartile range]

CCI Comprehensive Complication Index, ICU intensive care unit, IPTW inverse probability of treatment weighting, ISGLS International Study Group of Liver Surgery, $P H L F$ post-hepatectomy liver failure, $P R B C$ packed red blood cell, $S D$ standard deviation

${ }^{a} \mathrm{CCI}$ for entire group; for only patients that developed complications, CCI at discharge was $27.0 \pm 12.2 \mathrm{OHH}$ and $25.2 \pm 20.8 \mathrm{LHH}$ and at 90 days $28.4 \pm 12.0 \mathrm{OHH}$ and $24.8 \pm 18.8 \mathrm{LHH}$

${ }^{\mathrm{b}}$ Does not include ISGLS PHLF Grade A 
loss was less (300 [150-500] mL OHH, 200 [50-300] mL $\mathrm{LHH}, p=0.018)$. There were no differences in rates of " $\mathrm{R} 1$ " resection (tumor cells $\leq 1 \mathrm{~mm}$ from the transection margin) detected between the two groups: $14 \% \mathrm{OHH}$ and $11 \% \mathrm{LHH}$, $p=0.612$.

\section{Postoperative complications and outcomes}

Postoperative complications and events are described in Table 3. Consequent to less intraoperative blood loss, fewer patients undergoing $\mathrm{LHH}$ received perioperative blood transfusions $(15 \% \mathrm{OHH}, 1 \% \mathrm{LHH}, p=0.024)$. As well, fewer complications occurred in the LHH group prior to hospital discharge (overall 60\% OHH, 39\% LHH, $p=0.039$ ), and CCI measured up to discharge was also lower with LHH $(16.5 \pm 16.1 \mathrm{OHH}, 9.7 \pm 14.2, p=0.009)$. These differences were reflected in a reduction in ICU and overall postoperative hospital stays for patients in the LHH vs. OHH groups by a median of 2 days for each ( $p<0.001$ in both cases).

By 90 days after surgery, overall complication rates were $69 \% \mathrm{OHH}$ and $44 \% \mathrm{LHH}(p=0.01)$. Accordingly, $\mathrm{CCI}$ at 90 days was also lower with LHH $(20.0 \pm 16.1$ $\mathrm{OHH}, 10.9 \pm 14.2 \mathrm{LHH}, p=0.001)$. Upon examination of the specific complications that arose during 90 days, liver-specific complications, including bile leak, ascites, encephalopathy, and PHLF requiring a change in patient management, occurred at similar rates following both $\mathrm{OHH}$ and LHH. Surgical complications also occurred at relatively similar rates, though there was a tendency toward more postoperative hemorrhage and superficial surgical site infection among OHH patients. Medical complications, on the other hand, occurred at higher rates in the OHH group when compared with LHH (37\% OHH, 16\% LHH, $p=0.022$ ). Medical complications were primarily pulmonary complications, including pneumonias and pleural effusions, and complications associated with the use of indwelling catheters, such as urinary tract infections and bloodstream infections arising from central venous catheters.

Rates of surgical re-intervention during the first 90 days did not differ between the OHH and LHH groups (6\% and $5 \%$, respectively, $p=0.758$ ). Six patients undergoing $\mathrm{OHH}$ were re-operated: two each for hemorrhage and bile leak during the index admission and another two for bowel obstruction on a subsequent readmission. Two patients undergoing LHH were re-operated laparoscopically for bile leak during the index admission.

There was a greater tendency toward readmissions among patients undergoing $\mathrm{OHH}$ as opposed to LHH (17\% vs. $4 \%$, respectively), though this difference did not reach the level statistical significance $(p=0.055)$. In terms of mortality, there was only one death prior to 90 days in

Table 4 Cost analysis evaluated up to 90 days postoperatively

\begin{tabular}{|c|c|c|c|c|c|c|c|}
\hline & \multicolumn{3}{|l|}{ Raw analysis } & \multicolumn{4}{|l|}{ IPTW analysis } \\
\hline & $\mathrm{OHH}(n=96)$ & LHH $(n=45)$ & $p$ value & $\mathrm{OHH}$ & LHH & $\%$ difference & $p$ value \\
\hline \multicolumn{8}{|l|}{ Intraoperative costs } \\
\hline Consumables & 1801 [1468-2404] & 3374 [2898-3889] & $<0.001$ & 1777 [1429-2387] & 3253 [2958-3889] & +83 & $<0.001$ \\
\hline OR time & 1408 [1167-1748] & 1827 [1452-2224] & $<0.001$ & 1386 [1161-1745] & 1730 [1393-2225] & +29 & $<0.001$ \\
\hline OR staff & 821 [637-1262] & 1290 [953-1551] & $<0.001$ & 788 [632-1318] & 1264 [902-1548] & +60 & $<0.001$ \\
\hline Total & 4309 [3584-5348] & 7239 [5542-8244] & $<0.001$ & $4339[3541-5346]$ & 6917 [5314-8363] & +59 & $<0.001$ \\
\hline \multicolumn{8}{|l|}{ Postoperative costs } \\
\hline Pharmacy & 184 [50-297] & 100 [30-175] & 0.067 & 163 [39-274] & 100 [27-157] & -40 & 0.114 \\
\hline Transfusions & $90 \pm 162$ & $22 \pm 111$ & $<0.001$ & $80 \pm 153$ & $10 \pm 67$ & -88 & 0.001 \\
\hline Postoperative stay & 3896 [2603-4972] & 2070 [1648-3313] & $<0.001$ & 3574 [1978-4805] & 2046 [1560-3012] & -43 & $<0.001$ \\
\hline ICU & 2379 [1121-3311] & 797 [0-2212] & $<0.001$ & 2212 [0-3188] & 761 [0-1592] & -65 & $<0.001$ \\
\hline Surgical floor & 1492 [1183-1872] & 1111 [857-1533] & $<0.001$ & 1444 [1182-1850] & 1182 [887-1560] & -18 & 0.002 \\
\hline Techniques & 142 [67-339] & 87 [32-166] & 0.008 & 124 [53-302] & 60 [31-177] & -69 & 0.086 \\
\hline Miscellaneous $^{\mathrm{a}}$ & 707 [501-1117] & 515 [302-632] & $<0.001$ & 651 [455-1075] & 515 [294-580] & -21 & 0.002 \\
\hline Total & 4990 [3751-6434] & 2924 [2079-4449] & $<0.001$ & 4817 [2956-6251] & 2875 [2066-4109] & -40 & $<0.001$ \\
\hline $\begin{array}{l}\text { Total surgical } \\
\text { admission costs }\end{array}$ & 9916 [7766-12290] & $\begin{array}{l}10,448[7703- \\
13730]\end{array}$ & 0.270 & 9254 [7134-11908] & $\begin{array}{l}10,396[7540- \\
13730]\end{array}$ & +12 & 0.195 \\
\hline Readmission costs & $372 \pm 1043$ & $232 \pm 1195$ & 0.140 & $331 \pm 944$ & $110 \pm 699$ & -67 & 0.067 \\
\hline Total cost at 90 days & $\begin{array}{l}10,112 \text { [7849- } \\
12433]\end{array}$ & $\begin{array}{l}10,931 \text { [8374- } \\
14240]\end{array}$ & 0.300 & 9936 [7134-11923] & $\begin{array}{l}10,448 \text { [7703- } \\
14240]\end{array}$ & +5 & 0.286 \\
\hline
\end{tabular}

Values are reported in Euros $(€)$ and presented as mean \pm standard deviation or median [25-75\% interquartile range]

${ }^{a}$ Laboratory tests and medical care during floor stay 
the entire cohort: a patient that underwent LHH died of massive pulmonary embolism during the first postoperative week.

\section{Costs}

Perioperative costs are illustrated in Table 4. All operating room costs, including consumable material and costs associated with OR use and staffing, were higher for LHH versus OHH by a median of approximately $2500 €$ per procedure $(p<0.001)$. In contrast, postoperative costs, in particular those related to ICU and floor stays and medical staffing and care, were lower for LHH versus OHH by a median of roughly $2000 €$ per patient $(p<0.001)$. Costs for readmissions in Table 4 are presented as the median for all patients included in each group; costs for only cases where readmissions occurred were OHH (17 readmissions) $2049 €$ [1229-2411] and LHH 3 readmissions 1396€ [1229-4489], $p=1.0$. Considering that median readmission costs were also reduced by about $200 €$ among LHH patients (a difference that did not reach statistical significance, $p=0.067$ ), the overall balance of 90-day costs associated with $\mathrm{OHH}$ and LHH was similar: $9936 €[7134-11,908]$ and $10,448 €$ [7703-14,240], respectively, $p=0.286$.

\section{Discussion}

In this study, we meticulously evaluated complications and costs associated with hemihepatectomy arising between index procedures up to 90 days postoperatively. The major findings were a relatively high rate of largely medical complications following OHH that were concomitantly associated with increased ICU and overall postoperative hospital stays as well as higher postoperative costs relative to LHH. Readmission costs for $\mathrm{OHH}$ also tended to be higher. In contrast, upfront intraoperative costs associated with $\mathrm{OHH}$ were lower, and overall costs for the open and laparoscopic approaches to hemihepatectomy were equivalent after 3 months of follow-up.

Previous studies following hemihepatectomy patients during their index hospitalization up to the end of the first postoperative month have also found relative cost equity when comparing open and laparoscopic procedures [8-10]. It has been determined for major surgical interventions, however, that limiting the period of observation to the end of the first postoperative month may overlook significant further morbidity, mortality, and costs, and a 90-day observation period appears to more accurately capture all relevant postoperative events [11, 20-24]. In the present study, additional complications occurring beyond discharge were not negligible, with approximately $10 \%$ more complications arising between discharge and 90 days among OHH patients and 5\% more complications between discharge and 90 days among those undergoing LHH. Additional postoperative complications are not only relevant to patient satisfaction and quality of life but are also known to be a primary factor that serve to increase healthcare costs related to surgery $[25,26]$.

The overall rate of complications detected by 90 days in this study was ostensibly high: $69 \%$ for $\mathrm{OHH}$ and $44 \%$ for LHH. On the other hand, rates of moderate and severe complications requiring invasive therapy or management in intensive care (i.e., Clavien-Dindo III-V: 23\% OHH and $11 \% \mathrm{LHH}$ ) were relatively low in comparison with other recent studies examining outcomes following hemihepatectomy [6-10]. Moreover, among patients developing complications, CCI, which provides a comprehensive view of all postoperative complications, with each graded according to its clinical impact (i.e., resources required to treat) remained below benchmark values in both groups, both at discharge $(<27.9)$ and at 3 months $(<32.6)$ [11].

Liver-specific complications were similar between the two groups in this study, though there was a tendency towards a higher rate of grade A PHLF (ongoing abnormal laboratory values requiring no change in clinical management) following $\mathrm{OHH}$. Rates of surgical complications (intraabdominal abscess/fluid collection, mechanical intestinal obstruction, hemorrhage/hematoma, and superficial surgical site infection) were the same. However, rates of medical complications were significantly higher for $\mathrm{OHH}$.

Medical complications not reaching the point of requiring invasive and/or intensive care treatment may have been under-detected and/or -reported in previous retrospective studies evaluating outcomes following hemihepatectomy $[6,7,10,27]$. We exhaustively evaluated all postoperative events, including delay in return of normal bowel function, which was surprisingly not improved among patients undergoing LHH. Manipulation of the bowel in both LHH and even $\mathrm{OHH}$ remains limited with respect to other intraabdominal procedures, yet hemihepatectomy can significantly impact bowel function based on the aggressive nature of the procedure itself, splanchnic congestion developing secondary to significant reduction in the size of the portal sinusoidal bed, and a certain degree of portal hypertension developing in the postoperative period [28]. These events are the same regardless of the surgical approach. As well, due to inability to perform manual compression of the cut surface during transection, we frequently use the intermittent Pringle maneuver during laparoscopic hepatectomy to limit blood loss during parenchymal transection. For this reason, rates of utilization and overall length of hepatic hilar clamping were significantly greater for LHH than OHH. This, too, might have contributed to the $14 \%$ rate of postoperative paralytic ileus following LHH that we observed in our series. Given that return to normal bowel function is one of the key 
endpoints in some prospective trials aiming to determine the impact of the laparoscopic approach on "functional recovery" following surgery [29-31], this finding is noteworthy and a cause for reflection as to whether such an endpoint is the most appropriate to evaluate the benefits and impact of laparoscopy in the setting of this particular procedure or procedures of a similar nature.

Pulmonary complications are common following liver resection, arising in $>20 \%$ of cases in most series [32,33]. A French multicenter study specifically focused on pulmonary complications following major hepatic resection and detected a high rate for open procedures $(41 \%)$ that was significantly reduced following laparoscopic procedures (13\%) [34]. In this study, pulmonary complications (including pneumonia, pleural effusion, and pulmonary embolism) were detected in $19 \%$ of patients following $\mathrm{OHH}$ but only $2 \%$ of patients following LHH. Actual or perceived pain and other functional limitations associated with open incision and costal retraction in $\mathrm{OHH}$ contribute to suboptimal pulmonary hygiene in the postoperative period. Following laparoscopic and even hybrid hemihepatectomy; however, early abdominal wall pain and function are considerably improved, leading to better respiratory and earlier physical activity.

Medbery and colleagues introduced the concept of "value" into the armamentarium of the benefits offered by laparoscopic liver surgery - that quality (e.g., outcomes) can be improved while cutting costs [8]. Unlike the singular and largely nascent endpoints that are used in most surgical trials, value takes into account not only perioperative events but also more remote outcome measures, such as physical recovery, ongoing pain, incisional hernias, disease recurrence, and survival. Given that the outcome benefits that laparoscopy offers may only be compounded over time, LHH appears to offer a value advantage over OHH. Furthermore, the value of LHH is likely to improve further still as surgical expertise increases and upfront costs associated with the operative procedure progressively reduce due to shorter operative times and the ability to perform procedures with a more limited range of surgical instruments and devices. This is especially true for new generations of surgeons, who train directly in laparoscopy in other abdominal interventions (e.g., cholecystectomies, appendectomies, colectomies) and will also train directly in laparoscopic hepatectomies using standardized techniques honed by their predecessors.

The present study does have limitations related to its retrospective nature and the non-randomized distribution of patients. Inverse probability of treatment weighting is a propensity method that does not remove patients but gives them distinct weights based on over- or underrepresentation of certain influential characteristics. Following IPTW adjustments, the results of this study continue to support the raw and unadjusted findings. Nonetheless, even IPTW adjustments cannot achieve the same balanced distribution of unquantified or unidentified variables as in a randomized design [35]. Another limitation is the relatively small sample size, which is partially a consequence of increasing use of parenchymal-sparing surgical techniques, particularly for the resection of metastatic lesions [36]. As well, there is the fact that ten patients in the LHH group were operated using a hybrid approach. These patients belonged to the first 3 years of the inclusion period. It is important to note that all hybrid cases were planned preoperatively and were not unplanned conversions, which have been shown to be associated with greater perioperative morbidity and mortality [37]. Comparing the 35 pure laparoscopic with the ten hybrid hemihepatectomies, both CCI at discharge and 90 days and costs at discharge and 90 days tended to be lower for the hybrid procedures, by and large based on the fact that intraoperative costs for the planned hybrid procedures were lower (7260€ [5723-7988] and $4937 €$ [3473-6954], respectively, $p=0.024$ ).

In summary, while laparoscopic hemihepatectomy remains a complex surgical procedure, it appears to offer considerable benefits over the open approach in terms of morbidity and costs arising postoperatively, not only during index admission but also in subsequent readmissions. These savings in the postoperative period serve to offset the initial increase in operating room costs associated with laparoscopy. In the future, as experience with and results following minimally invasive liver surgery continue to improve, it is plausible that the laparoscopic or other minimally invasive approaches may eventually become the standard-of-care for hemihepatectomy as they already have for other more minor forms of liver resection.

Author contributions Conception and design: FR, CM, FA, AJH, and CF. Provision of study materials or patients: DC, RGP, JF, JF, JCGV, and CF. Collection and assembly of data: FR, CM, and RS. Data analysis and interpretation: FR, FA, AJH, and CF. Manuscript writing: all authors. Final approval of manuscript: all authors.

\section{Funding None.}

\section{Compliance with ethical standards}

Conflict of interest F. Riquelme, C. Muñoz, F. Ausania, F. Torres, D. Calatayud, R. Sandomenico, R. García Péres, J. Ferrer, J. Fuster, and J.C. García-Valdecasas have no conflicts of interest to disclose. A.J. Hessheimer and C. Fondevila are Principal Investigators on a Fondo de Investigación Sanitaria Grant (PI1800894) from the Spanish Ministry of Health.

Ethical approval This is stated in Methods (part on "Study Design"): Approval for the study was obtained from our Institution's Committee on Ethics in Medical Research prior to initiating data analysis (study number HCB/2018/1179), which waived the need for written consent. 
Informed consent Given the nature of the study, informed consent was waived by the Institutional Review Board.

Open Access This article is licensed under a Creative Commons Attribution 4.0 International License, which permits use, sharing, adaptation, distribution and reproduction in any medium or format, as long as you give appropriate credit to the original author(s) and the source, provide a link to the Creative Commons licence, and indicate if changes were made. The images or other third party material in this article are included in the article's Creative Commons licence, unless indicated otherwise in a credit line to the material. If material is not included in the article's Creative Commons licence and your intended use is not permitted by statutory regulation or exceeds the permitted use, you will need to obtain permission directly from the copyright holder. To view a copy of this licence, visit http://creativecommons.org/licenses/by/4.0/.

\section{References}

1. Bhojani FD, Fox A, Pitzul K, Gallinger S, Wei A, Moulton CA et al (2012) Clinical and economic comparison of laparoscopic to open liver resections using a 2-to-1 matched pair analysis: an institutional experience. J Am Coll Surg 214(2):184-195

2. Ciria R, Cherqui D, Geller DA, Briceno J, Wakabayashi G (2016) Comparative short-term benefits of laparoscopic liver resection: 9000 cases and climbing. Ann Surg 263(4):761-777

3. Fretland AA, Dagenborg VJ, Bjornelv GMW, Kazaryan AM, Kristiansen R, Fagerland MW et al (2018) Laparoscopic versus open resection for colorectal liver metastases: the OSLOCOMET randomized controlled trial. Ann Surg 267(2):199-207

4. Macacari RL, Coelho FF, Bernardo WM, Kruger JAP, Jeismann VB, Fonseca GM et al (2019) Laparoscopic vs. open left lateral sectionectomy: an update meta-analysis of randomized and nonrandomized controlled trials. Int J Surg 61:1-10

5. Abu HM, Aldrighetti L, Dagher I, Edwin B, Troisi RI, Alikhanov R et al (2018) The southampton consensus guidelines for laparoscopic liver surgery: from indication to implementation. Ann Surg 268(1):11-18

6. van der Poel MJ, Besselink MG, Cipriani F, Armstrong T, Takhar AS, Van DS et al (2016) Outcome and learning curve in 159 consecutive patients undergoing total laparoscopic hemihepatectomy. JAMA Surg 151(10):923-928

7. Abu HM, Di FF, Syed S, Wiltshire R, Dimovska E, Turner D et al (2013) Assessment of the financial implications for laparoscopic liver surgery: a single-centre UK cost analysis for minor and major hepatectomy. Surg Endosc 27(7):2542-2550

8. Medbery RL, Chadid TS, Sweeney JF, Knechtle SJ, Kooby DA, Maithel SK et al (2014) Laparoscopic vs open right hepatectomy: a value-based analysis. J Am Coll Surg 218(5):929-939

9. Cipriani F, Ratti F, Cardella A, Catena M, Paganelli M, Aldrighetti L (2019) Laparoscopic versus open major hepatectomy: analysis of clinical outcomes and cost effectiveness in a highvolume center. J Gastrointest Surg 23(11):2163-2173

10. Wabitsch S, Kastner A, Haber PK, Feldbrugge L, Winklmann $\mathrm{T}$, Werner $\mathrm{S}$ et al (2019) Laparoscopic versus open hemihepatectomy - a cost analysis after propensity score matching. Langenbecks Arch Surg 404(4):469-475

11. Rossler F, Sapisochin G, Song G, Lin YH, Simpson MA, Hasegawa K et al (2016) Defining benchmarks for major liver surgery: a multicenter analysis of 5202 living liver donors. Ann Surg 264(3):492-500

12. Majno PE, Mentha G, Morel P, Segalin A, Azoulay D, Oberholzer J et al (2002) Arantius' ligament approach to the left hepatic vein and to the common trunk. J Am Coll Surg 195(5):737-739

13. Charlson M, Szatrowski TP, Peterson J, Gold J (1994) Validation of a combined comorbidity index. J Clin Epidemiol 47(11):1245-1251

14. Dindo D, Demartines N, Clavien PA (2004) Classification of surgical complications: a new proposal with evaluation in a cohort of 6336 patients and results of a survey. Ann Surg 240(2):205-213

15. Slankamenac K, Graf R, Barkun J, Puhan MA, Clavien PA (2013) The comprehensive complication index: a novel continuous scale to measure surgical morbidity. Ann Surg 258(1):1-7

16. Koch M, Garden OJ, Padbury R, Rahbari NN, Adam R, Capussotti L et al (2011) Bile leakage after hepatobiliary and pancreatic surgery: a definition and grading of severity by the International Study Group of Liver Surgery. Surgery 149(5):680-688

17. Rahbari NN, Garden OJ, Padbury R, Brooke-Smith M, Crawford M, Adam R et al (2011) Posthepatectomy liver failure: a definition and grading by the International Study Group of Liver Surgery (ISGLS). Surgery 149(5):713-724

18. Ishizawa T, Hasegawa K, Kokudo N, Sano K, Imamura H, Beck $\mathrm{Y}$ et al (2009) Risk factors and management of ascites after liver resection to treat hepatocellular carcinoma. Arch Surg 144(1):46-51

19. Instituto Nacional de Estadística (Spain) (2016) Índices de Precios de Consumo. Base. Available from: https://www.ine.es/jaxiT3/ Tabla.htm? $\mathrm{t}=22350 \& \mathrm{~L}=0$.

20. Pezzi CM, Mallin K, Mendez AS, Greer GE, Putnam JB Jr (2014) Ninety-day mortality after resection for lung cancer is nearly double 30-day mortality. J Thorac Cardiovasc Surg 148(5):2269-2277

21. Swanson RS, Pezzi CM, Mallin K, Loomis AM, Winchester DP (2014) The 90-day mortality after pancreatectomy for cancer is double the 30-day mortality: more than 20,000 resections from the national cancer data base. Ann Surg Oncol 21(13):4059-4067

22. Talsma AK, Lingsma HF, Steyerberg EW, Wijnhoven BP, Van Lanschot JJ (2014) The 30-day versus in-hospital and 90-day mortality after esophagectomy as indicators for quality of care. Ann Surg 260(2):267-273

23. Mise Y, Vauthey JN, Zimmitti G, Parker NH, Conrad C, Aloia TA et al (2015) Ninety-day postoperative mortality is a legitimate measure of hepatopancreatobiliary surgical quality. Ann Surg 262(6):1071-1078

24. Schiergens TS, Dorsch M, Mittermeier L, Brand K, Kuchenhoff $\mathrm{H}$, Lee SM et al (2015) Thirty-day mortality leads to underestimation of postoperative death after liver resection: a novel method to define the acute postoperative period. Surgery 158(6):1530-1537

25. Vonlanthen R, Slankamenac K, Breitenstein S, Puhan MA, Muller MK, Hahnloser D et al (2011) The impact of complications on costs of major surgical procedures: a cost analysis of 1200 patients. Ann Surg 254(6):907-913

26. Staiger RD, Cimino M, Javed A, Biondo S, Fondevila C, Perinel $\mathrm{J}$ et al (2018) The Comprehensive Complication Index (CCI(R)) is a novel cost assessment tool for surgical procedures. Ann Surg 268(5):784-791

27. Cipriani F, Alzoubi M, Fuks D, Ratti F, Kawai T, Berardi G et al (2020) Pure laparoscopic versus open hemihepatectomy: a critical assessment and realistic expectations-a propensity score-based analysis of right and left hemihepatectomies from nine European tertiary referral centers. J Hepatobiliary Pancreat Sci 27(1):3-15

28. Hessheimer AJ, de la Martinez ML, Adel Al SF, Espinoza AS, Ausania F, Fondevila C (2019) Somatostatin and the "small-forsize" liver. Int J Mol Sci 20(10):2512

29. Wong-Lun-Hing EM, van Dam RM, van Breukelen GJ, Tanis PJ, Ratti F, Van HR et al (2017) Randomized clinical trial of open versus laparoscopic left lateral hepatic sectionectomy within an enhanced recovery after surgery programme (ORANGE II study). Br J Surg 104(5):525-535 
30. ClinicalTrials.gov. Bethesda (MD): National Library of Medicine (US). 2011 Sep 28. Identifier NCT01441856, The ORANGE II PLUS - Trial: Open Versus Laparoscopic Hemihepatectomy. Available from: https://clinicaltrials.gov/ct2/show/NCT01441856.

31. ClinicalTrials.gov. Bethesda (MD): National Library of Medicine (US). 2017 Sep 1. Identifier NCT03270917, ORANGE SEGMENTS: Open Versus Laparoscopic Parenchymal Preserving Postero-Superior Liver Segment Resection. Available from: https ://clinicaltrials.gov/ct2/show/NCT03270917.

32. Yang T, Zhang J, Lu JH, Yang GS, Wu MC, Yu WF (2011) Risk factors influencing postoperative outcomes of major hepatic resection of hepatocellular carcinoma for patients with underlying liver diseases. World J Surg 35(9):2073-2082

33. Nobili C, Marzano E, Oussoultzoglou E, Rosso E, Addeo P, Bachellier P et al (2012) Multivariate analysis of risk factors for pulmonary complications after hepatic resection. Ann Surg 255(3):540-550

34. Fuks D, Cauchy F, Fteriche S, Nomi T, Schwarz L, Dokmak S et al (2016) Laparoscopy decreases pulmonary complications in patients undergoing major liver resection: a propensity score analysis. Ann Surg 263(2):353-361
35. Torres F, Rios J, Saez-Penataro J, Pontes C (2017) Is propensity score analysis a valid surrogate of randomization for the avoidance of allocation bias? Semin Liver Dis 37(3):275-286

36. Moris D, Dimitroulis D, Vernadakis S, Papalampros A, Spartalis E, Petrou A et al (2017) Parenchymal-sparing hepatectomy as the new doctrine in the treatment of liver-metastatic colorectal disease: beyond oncological outcomes. Anticancer Res 37(1):9-14

37. Halls MC, Cipriani F, Berardi G, Barkhatov L, Lainas P, Alzoubi $\mathrm{M}$ et al (2018) Conversion for unfavorable intraoperative events results in significantly worse outcomes during laparoscopic liver resection: lessons learned from a multicenter review of 2861 cases. Ann Surg 268(6):1051-1057

Publisher's Note Springer Nature remains neutral with regard to jurisdictional claims in published maps and institutional affiliations. 